\title{
EFEITO DE Diferentes PrÉ-TRATAMENTOS DE CULTURA MISTA PARA O ENRIQUECIMENTO EM BACTÉRIAS PRODUTORAS DE HIDROGÊNIO
}

\begin{abstract}
RESUMO - Neste trabalho foi estudado o efeito de diferentes pré-tratamentos sobre uma cultura mista coletada de um sistema anaeróbio de tratamento de vinhaça com relação à produção de $\mathrm{H}_{2}$. Foram realizados os seguintes pré-tratamentos: 1) Tratamento térmico a $100^{\circ} \mathrm{C}$ por $15 \mathrm{~min}$.; 2) Tratamento térmico a $100^{\circ} \mathrm{C}$ por $60 \mathrm{~min}$; 3) Tratamento térmico a $80^{\circ} \mathrm{C}$ por $15 \mathrm{~min}$; 4) Tratamento térmico a $80^{\circ} \mathrm{C}$ por $60 \mathrm{~min}$; 5) Tratamento ácido ( $\mathrm{pH} 3$ com $\mathrm{HCl}$ ); 6) Tratamento combinado ( $\mathrm{pH} 3$ e fervura por 30 min.); 7 e 8) Lavagem seletiva do lodo em reator contínuo, operado a $50^{\circ} \mathrm{C}$ com TRH de $12 \mathrm{~h}$ e $6 \mathrm{~h}$. O modelo cinético de Gompertz modificado foi utilizado para a obtenção dos parâmetros cinéticos. As maiores velocidades de produção $(\mathrm{Rm})$ foram observadas com o lodo que sofreu pré-tratamento térmico $80^{\circ} \mathrm{C}$ por $15 \mathrm{~min}$; t térmico $80^{\circ} \mathrm{C}$ por $60 \mathrm{~min}$. e ácido $\mathrm{pH} 3$ por 24h; 12,2; 15,6 e 16,5 mL/h, respectivamente. Em ensaios de longa duração (por 36 dias), o tratamento ácido demonstrou ser o mais efetivo para o enriquecimento da cultura mista para a produção de $\mathrm{H}_{2}$.
\end{abstract}

\section{1- INTRODUÇÃO}

Um dos maiores desafios para a produção biológica de $\mathrm{H}_{2}$ é a preparação de grandes quantidades de fontes naturais de inóculo estáveis e ativos. Para aumentar a velocidade de produção de $\mathrm{H}_{2}$ é importante desenvolver métodos que inibam a atividade das bactérias consumidoras de $\mathrm{H}_{2}$, tais como as metanogênicas, desnitrificantes e redutoras de sulfato, e que favoreçam o enriquecimento das bactérias produtoras de $\mathrm{H}_{2}$, tais como as dos gêneros Clostridium e Enterobacter (Hawkes et al., 2002).

Muitos são os métodos de pré-tratamento descritos na literatura para o enriquecimento de bactérias produtoras de $\mathrm{H}_{2}$. Dentre eles destacam-se o térmico (Lay et al., 2012) o químico com ácidos ou bases (Asswamonghlosiri et al., 2013) e a lavagem seletiva (Luo et al., 2010).

A grande maioria dos artigos sobre a produção biológica de $\mathrm{H}_{2}$ utiliza lodos anaeróbios, pré-tratados termicamente (Lay et al., 2012). O tratamento térmico do lodo cria condições adversas aos micro-organismos não esporulantes, portanto é comumente utilizado para o enriquecimento em bactérias formadoras de esporos, como o Clostridium, principal gênero produtor de $\mathrm{H}_{2}$.

Condições ácidas ou básicas são recomendadas para tratar lodos e aumentar a produção de $\mathrm{H}_{2}$, por inibirem bactérias sensíveis a valores de $\mathrm{pHs}$ extremos (Asswamonghlosiri et al., 2013).

A lavagem seletiva do lodo é uma estratégia utilizada com a finalidade de selecionar, em reatores contínuos, os microrganismos mais especializados e ativos nas condições de operação do reator. Esta estratégia tem como fundamento a utilização de tempos de retenção hidráulico (TRHs) mais baixos do que as velocidades específicas de crescimento das bactérias que se pretende lavar, de forma a tornar o lodo mais rico nas bactérias de interesse (Luo et al., 2010). 
Mohan (2009), em revisão de literatura aponta para a necessidade do pré-tratamento de culturas mistas a fim de enriquecer o inóculo em bactérias acidogênicas/acetogênicas e revela elevadas diferenças de rendimentos de produção de $\mathrm{H}_{2}$ entre lodos pré-tratados e não tratados obtidos por diferentes autores. Entretanto, este mesmo autor alerta que cada método de pré-tratamento tem a sua eficácia associada à natureza do inóculo, ao substrato e a forma de operação do biorreator. Neste sentido, o efeito do tratamento do inóculo na produção de $\mathrm{H}_{2}$ deve ser estudado para cada combinação de cultura mista e substrato.

Entretanto, com relação ao uso de pré-tratamentos para o enriquecimento em bactérias produtoras de $\mathrm{H}_{2}$, Luo et al., (2010) alertam para os riscos em se extrapolar os resultados do aumento da produção de $\mathrm{H}_{2}$ observado em culturas mistas pré-tratadas em testes em batelada, para o processo contínuo, operado por longos períodos. Pois, a longo prazo estes resultados podem não se manter e as condições impostas aos biorreatores podem resultar no estabelecimentos de outras comunidades microbianas.

Neste trabalho, foram estudados os efeitos de curto e longo prazo de diferentes prétratamentos de cultura mista utilizada no tratamento de vinhaça sobre a produção biológica de $\mathrm{H}_{2}$.

\section{MATERIAIS E MÉTODOS}

\subsection{Inóculo e Meio de Fermentação}

A cultura mista (lodo) utilizada como inóculo foi coletada de um reator anaeróbio de fluxo ascendente para o tratamento de efluentes de uma usina de açúcar e etanol (vinhaça), localizada na Região de Ribeirão Preto - SP. O lodo foi mantido em laboratório pela sua alimentação com meio de cultura contendo glicose como fonte de carbono, e a composição de nutrientes adaptado de Del Nery (1987). Todos os produtos químicos eram de grau analítico. Para a caracterização do inóculo foi realizada a análise de sólidos voláteis (SV) no lodo seco, de acordo com APHA (1995).

\subsection{Pré-tratamentos do inóculo}

Foram utilizados oito métodos de pré-tratamento do inóculo. Os tratamentos térmicos do inóculo foram realizados em um reator de vidro com volume de 1,5 $\mathrm{L}$ ao qual foi adicionado $1 \mathrm{~L}$ do lodo no reator e submetidos à temperatura em banho termostatizado. As condições de tempo e temperatura foram de $100^{\circ} \mathrm{C}$ por $15 \mathrm{~min}$. e $60 \mathrm{~min}$., $80^{\circ} \mathrm{C}$ por $15 \mathrm{~min}$. e por $60 \mathrm{~min}$. Todos os pré-tratamentos térmicos foram seguidos de resfriamento rápido (banho de gelo). O pré-tratamento ácido do inóculo foi realizado em um reator com volume de 1,5 $\mathrm{L}$ ao qual foi adicionado $1 \mathrm{~L}$ do lodo no reator e acidificado até $\mathrm{pH} 3,0 \mathrm{com} \mathrm{HCl} 1$ $\mathrm{mol} / \mathrm{L}$, permanecendo neste $\mathrm{pH}$ por $24 \mathrm{~h}$. O tratamento combinado foi realizado com o lodo aciidificado pela sua fervura por $30 \mathrm{~min}$. Para a lavagem do lodo foi utilizado um biorreator de vidro de volume de $1,5 \mathrm{~L}$, mantido a $50^{\circ} \mathrm{C}$ e inoculado com $1 \mathrm{~L}$ de lodo. A concentração de sólidos voláteis totais inicial foi determinada, em seguida iniciou-se a alimentação contínua do reator que foi operado como um reator contínuo com Tempo de Retenção Hidráulico (TRH) de 12 e 6 h.

Após a realização dos pré-tratamentos, os lodos foram utilizados como inóculo para ensaios cinéticos de produção de $\mathrm{H}_{2}$ em batelada. 


\subsection{Ensaios de Produção de $\mathrm{H}_{2}$ em batelada}

Foram realizados ensaios em batelada para a produção de $\mathrm{H}_{2}$ utilizando a cultura mista de microrganismos (lodo) sem pré-tratamento (controle) e após os pré-tratamentos. Os ensaios foram realizados em biorreatores de $450 \mathrm{~mL}$, adicionando-se $300 \mathrm{~mL}$ de lodo e 150 $\mathrm{mL}$ de meio de cultura (Del Nery, 1987), contendo $5 \mathrm{~g} / \mathrm{L}$ de glicose e cerca de $2,4 \mathrm{~g} / \mathrm{L}$ de SV. O $\mathrm{pH}$ inicial no biorreator foi ajustado para $6,5( \pm 0,2)$ e a condição anaeróbia mantida pelo borbulhamento de gás argônio durante $2 \mathrm{~min}$. Os ensaios forma realizados em triplicata.

O sistema operacional do reatores em batelada foi montado no interior de uma estufa, onde a temperatura foi mantida a $50^{\circ} \mathrm{C}$ e a agitação orbital em $200 \mathrm{rpm}$. A tampa do biorreator é constituída por rolha de silicone, na qual são acopladas duas tubulações: uma para a entrada de gás argônio (remoção de ar) e retirada de amostra e outra para saída do gás produzido no sistema. A tubulação de saída de gás foi acoplada a um sistema, no qual o gás produzido é recolhido em um frasco tipo Mariotte invertido contendo $\mathrm{NaOH} 5 \%(\mathrm{~m} / \mathrm{v})$. O volume de $\mathrm{NaOH}$ deslocado é captado em uma proveta e este representa o volume total de gás gerado, exceto o $\mathrm{CO}_{2}$ que fica retido no $\mathrm{NaOH}$. A composição do gás gerado foi analisada por cromatografia a gás. $\mathrm{E}$ o volume de $\mathrm{H}_{2}$ foi obtido multiplicando-se o volume total do gás pela percentagem de $\mathrm{H}_{2}$ analisada no gás.

\subsection{Modelagem dos resultados experimentais de produção de $\mathbf{H 2}$}

O modelo de Gompertz modificado (Ning et al., 2012), foi utilizado para os ensaios cinéticos de produção de $\mathrm{H}_{2}$ foi o qual relaciona três parâmetros $\mathrm{Rm}, \mathrm{P}$ e $\lambda$, conforme Equação 1. Os resultados de volume de $\mathrm{H}_{2}$ acumulado em função do tempo foram colocados no programa Statistica 7 e modelados pela equação 1.

$$
P=H_{\max }{ }^{*} \exp \left\{-\exp \left\{R m^{\left.\left.\left.\ell / H_{\max }\right)^{*}(\lambda-t)+1\right]\right\}}\right.\right.
$$

Onde: P: representa a produção de $\mathrm{H}_{2}$ acumulado $(\mathrm{mL})$; Hmax: potencial máximo de produção de $\mathrm{H}_{2}(\mathrm{~mL}) ; \mathrm{Rm}$ : velocidade máxima de produção de $\mathrm{H}_{2}(\mathrm{~mL} / \mathrm{h}) ; \lambda$ : tempo da fase lag (h) ou tempo para o início da produção de $\mathrm{H}_{2}$; t: tempo do experimento (h).

\subsection{Ensaios em batelada alimentada}

Após os ensaios em batelada foram escolhidos os pré-tratamentos que promoveram a maior produção de $\mathrm{H}_{2}$ para serem utilizados como inóculo em biorreatores operados em batelada alimentada por um período de 36 dias, em triplicata. Foi utilizada uma concentração de SV de 2,4 g.L-1 e os reatores foram operados retirando-se diariamente300 VmL do sobrenadante e alimentando-se com o mesmo volume de meio contendo $5 \mathrm{~g} / \mathrm{L}$ de glicose. $\mathrm{O}$ pH do meio de alimentação foi 6,5 e a temperatura mantida em $50^{\circ} \mathrm{C}$.

\subsection{Métodos Analíticos}

As determinações dos Sólidos Totais e Sólidos Voláteis (ST e SV) foram realizadas segundo APHA (2005).

A determinação da composição dos gases do headspace dos biorreatores foi realizada em cromatógrafo a gás CG 2014 da Shimadzu (Japão), equipado com detector de 
condutividade térmica (TCD). A coluna utilizada foi a peneira molecular 5A $2 \mathrm{~m}$ x $4,7 \mathrm{~mm}$, sendo o gás de arraste argônio sob vazão de $30 \mathrm{~mL} / \mathrm{min}$. As temperaturas do injetor, da coluna e do detector foram $80^{\circ} \mathrm{C}, 50^{\circ} \mathrm{C}$ e $100^{\circ} \mathrm{C}$, respectivamente.

A análise quantitativa da glicose e dos metabólitos solúveis da fermentação, tais como os ácidos orgânicos e etanol foi realizada por cromatografia líquida de alta eficiênciaCLAE- com detector de índice de refração, utilizando coluna Aminex HPX-87H, temperatura de $40^{\circ} \mathrm{C}$ e ácido sulfúrico a $1 \mathrm{mmol} / \mathrm{L}$ como fase móvel.

\section{RESULTADOS E DISCUSSÕES}

Na Figura 1 podem ser observados os volumes de $\mathrm{H}_{2}$ produzidos em função do tempo nos ensaios de fermentação em batelada com os inóculos pré-tratados e o controle.

Como pode ser observado na Figura 1 o lodo controle teve a sua produção de gás estabilizada antes dos lodos pré-tratados, em cerca de 18 horas de ensaio. Os lodos prétratados continuaram a produção de gás por um período mais prolongado.

A importância dos pré-tratamentos como métodos de enriquecimento do lodo para a produção de $\mathrm{H}_{2}$ pode ser verificada pelos resultados apresentados nesta figura, uma vez que os volumes de $\mathrm{H}_{2}$ produzidos pelos lodos que sofreram os pré-tratamentos de 1 a 6 foram superiores, quando comparados ao controle.

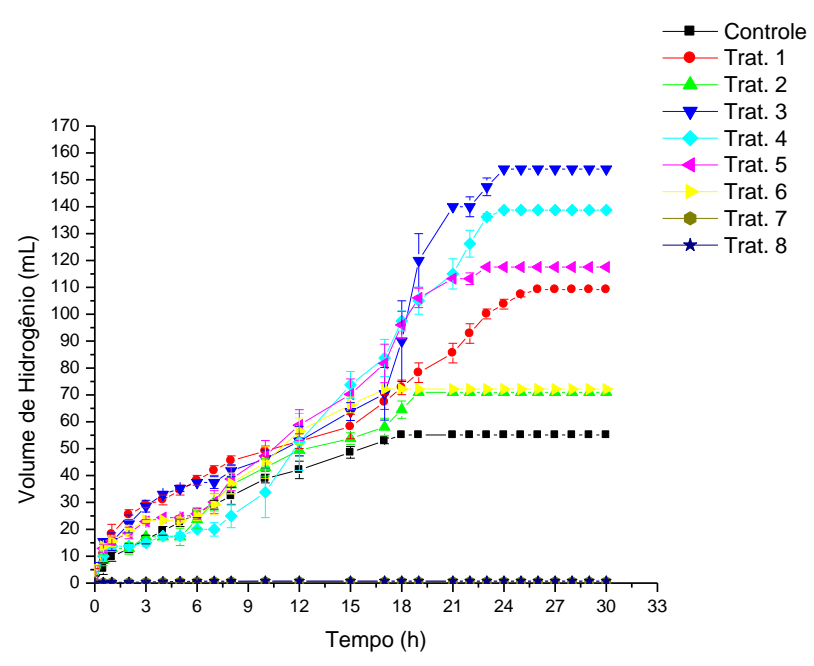

Figura 1 Volume de $\mathrm{H}_{2}$ produzido pelos lodos pré-tratados em função do tempo nos ensaios em batelada. Controle (sem pré-tratamento); 1- Térmico $100^{\circ} \mathrm{C} / 15 \mathrm{~min}$; 2- Térmico $100^{\circ} \mathrm{C} / 60$ min.; 3- Térmico $80^{\circ} \mathrm{C} / 15$ min.; 4- Térmico $80^{\circ} \mathrm{C} / 60 \mathrm{~min}$.; 5-Ácido pH 3/24h; 6Ácido pH 3/24h e fervura/30 min.; 7-Lavagem TRH 12h; 8-Lavagem TRH 6h.

Na Tabela 1 estão apresentados os parâmetros cinéticos obtidos a partir do modelo de Gompertz modificado para os ensaios em batelada com os lodos pré-tratados. Os resultados apresentados na Tabela 1 revelam que a maioria dos pré-tratamentos realizados na cultura mista foram efetivos para o enriquecimento em bactérias produtoras de $\mathrm{H}_{2}$, pois a produção máxima e a velocidade de produção aumentaram em relação ao controle. Apenas os lodos que sofreram lavagem seletiva (tratamentos 7 e 8 ) apresentaram produção máxima e 
velocidade de produção de $\mathrm{H}_{2}$ bem menores (praticamente nula), comparadas ao controle, sugerindo, que estes tratamentos não foram eficientes para enriquecer o lodo em bactérias produtoras de $\mathrm{H}_{2}$.

Tabela 1 Parâmetros cinéticos obtidos pelo modelo de Gompertz modificado para a produção de $\mathrm{H}_{2}$ nos ensaios com os lodos submetidos aos pré-tratamentos e o controle. (1Térmico $100^{\circ} \mathrm{C} / 15$ min.; 2- Térmico $100^{\circ} \mathrm{C} / 60 \mathrm{~min}$.; 3- Térmico $80^{\circ} \mathrm{C} / 15 \mathrm{~min}$.; 4- Térmico $80^{\circ} \mathrm{C} / 60$ min.; 5-Ácido pH 3/24h; 6- Ácido pH 3/24h e fervura/30 min.; 7-Lavagem TRH 12h; 8-Lavagem TRH 6h).

\begin{tabular}{lllll}
\hline & $\mathrm{Hmax}(\mathrm{mL})$ & $\mathrm{Rm}(\mathrm{mL} / \mathrm{h})$ & $\lambda(\mathrm{h})$ & $\mathrm{R}^{2}$ \\
\hline Controle & 31,2 & 3,22 & 1,17 & 0,998 \\
1 & 121,3 & 7,22 & 7,36 & 0,9834 \\
2 & 75,8 & 3,97 & 2,29 & 0,9942 \\
3 & 165,5 & 12,18 & 5,17 & 0,9916 \\
4 & 146,69 & 15,63 & 13,44 & 0,9934 \\
5 & 121,13 & 16,50 & 13,38 & 0,9968 \\
6 & 76,32 & 5,08 & 3,06 & 0,9866 \\
7 & 0,60 & 0,13 & $-0,42$ & 0,9921 \\
8 & 0,75 & 0,17 & $-0,36$ & 0,9938 \\
\hline
\end{tabular}

Hmax: potencial da produção máxima de $\mathrm{H}_{2}(\mathrm{~mL})$; Rm: velocidade máxima de produção de $\mathrm{H} 2(\mathrm{~mL} / \mathrm{h}) ; \lambda$ : fase lag $(\mathrm{h})$.

Em todos os testes realizados com os inóculos que sofreram pré-tratamentos efetivos para aumentar a produção de $\mathrm{H}_{2}$ (tratamentos de 1 a 6 ) foi observado um aumento do tempo necessário para o início da produção de $\mathrm{H}_{2}$, denominado de lag fase $(\lambda)$. Este efeito foi observado por outros autores, como por exemplo, Cheng et al., (2011) que utilizaram o tratamento térmico $\left(100^{\circ} \mathrm{C} / 30 \mathrm{~min}\right)$ e a fase lag do experimento aumentou 1,5 vezes comparada ao controle. A temperatura de $100^{\circ} \mathrm{C}$ (tratamentos 1 e 2) apesar de ser bastante citada na literatura como um método de enriquecimento em bactérias produtoras de $\mathrm{H}_{2}$, não foi a mais adequada para o pré-tratamento desta cultura mista. Esta temperatura e o tempo parecem ter sido muito elevados e podem ter causado a eliminação de microrganismos produtores de $\mathrm{H}_{2}$ não esporulados, tais como as enterobactérias.

De acordo com os resultados apresentados na Tabela 1, os pré-tratamentos do lodo que foram mais adequados para aumentar a velocidade de produção de $\mathrm{H}_{2}$ foram os prétratamentos 3, 4 e 5, ou seja; os tratamentos térmicos a $80^{\circ} \mathrm{C}$ por $15 \mathrm{~min}$. e por $30 \mathrm{~min}$. e o tratamento ácido $(\mathrm{pH} 3 / \mathrm{HCl})$, que apresentaram velocidades de 12,18, 15,63 e 16,50 ml.h ${ }^{-1}$, respectivamente.

As velocidades de produção de $\mathrm{H}_{2}$ encontradas na literatura variam muito, pois dependem do tipo de inóculo e os tipos de pré-tratamentos utilizados para o enriquecimento da cultura mista. Buitrón e Carvajal (2010) encontraram velocidades de produção de $\mathrm{H}_{2}$ que variaram entre 17,2 e $274 \mathrm{~mL} / \mathrm{h}$ e os valores da fase lag variaram de 0,5 a $1,7 \mathrm{~h}^{-1}$. Assawamongkholsiri et al., (2013) relatam velocidades de produção de $\mathrm{H}_{2}$ que variaram de 3,23 a $34,56 \mathrm{~mL} / \mathrm{h}$ e valores de fase lag entre 7,4 a $89,37 \mathrm{~h}^{-1}$, com inóculos que sofreram diferentes pré-tratamentos. 
O tempo e a temperatura utilizada para o tratamento térmico também difere bastante entre os trabalhos, sendo que $100^{\circ} \mathrm{C}$ entre 15 e 30 min. é a mais comum combinação (Chen et al., 2012). Muitos estudos têm utilizado diversas razões de tempo e temperatura para o pré-tratamento do lodo. De acordo com Li e Fang, (2009) não há condições ótimas de temperatura de aquecimento e duração do tratamento térmico, portanto, esta combinação deve ser estabelecida para cada lodo estudado.

Resultados similares aos deste trabalho também foram observados por outros autores. Lin et al.,(2006) submeteram uma cultura a um tratamento térmico com quatro temperaturas diferentes. $\mathrm{O}$ tratamento térmico a 50 e $60^{\circ} \mathrm{C}$ não foi eficiente para aumentar o desempenho da cultura mista na produção de $\mathrm{H}_{2}$. Porém, o tratamento térmico a 70 e $80^{\circ} \mathrm{C}$ aumentou significativamente o conteúdo de $\mathrm{H}_{2}$ no biogás. Jia et al., (2013) compara diferentes prétratamentos, como: ácido, alcalino, clorofórmio, 2-bromoetanosulfonato-BES, aeração, secagem, ultrassom e conclui que o pré-tratamento mais eficiente para aumentar a produção de $\mathrm{H}_{2}$ é a secagem do lodo a $60^{\circ} \mathrm{C}$ por $24 \mathrm{~h}$. Porém, Chang et al., (2011) avaliaram diferentes pré-tratamentos em lodo ativado para aumentar a produção de $\mathrm{H}_{2}$. Os pré-tratamentos utilizados pelos autores foram ácido, básico, choque térmico, aeração, uso de clorofórmio e BES. Neste estudo o lodo ativado pôde ser enriquecido por diferentes pré-tratamentos em ordem decrescentes de produção de $\mathrm{H}_{2}$ : ácido > base > térmico > aeração > clorofórmio > controle $>$ BES. Portanto, a maior quantidade de $\mathrm{H}_{2}$ produzida foi obtida pelo tratamento ácido, assim como em nosso trabalho.

Com relação à lavagem de bactérias, pode-se sugerir que os TRHs aplicados não foram efetivos para o enriquecimento em bactérias produtoras de $\mathrm{H}_{2}$, apesar de serem TRHs normalmente aplicados na literatura com sucesso para o enriquecimento de tais bactérias. Chen e Lin (2004) operaram um reator com sacarose para produzir $\mathrm{H}_{2}$, sem adaptação do inóculo, portanto com presença de bactérias consumidoras de $\mathrm{H}_{2}$. Com a diminuição do TRH de 20 para 2,5 d chegando a $6 \mathrm{~h}$, os autores conseguiram selecionar uma cultura denominada Clostridium pasteurianum, notadamente uma espécie produtora de $\mathrm{H}_{2}$.

Os resultados apresentados neste trabalho demonstram que diferentes métodos de prétratamento aplicados à cultura mista responderam de forma diferente, mas que os prétratamentos aumentaram a velocidade de produção de $\mathrm{H}_{2}$ entre 0,23 e 5,12 vezes quando comparado ao controle. Esta diferença entre as respostas obtidas pelos diferentes prétratamentos demonstra a importância do estudo de pré-tratamentos de inóculo para a produção de $\mathrm{H}_{2}$ para cada cultura mista estudada. Os resultados mostraram que os métodos que mais aumentaram a produção de $\mathrm{H}_{2}$ foi o 3,4 e 5 , ou seja, os tratamentos térmicos a $80^{\circ} \mathrm{C}$ por 15 min e 30 min. e o ácido $\mathrm{pH} 3$ por $24 \mathrm{~h}$, respectivamente.

Após os ensaios em batelada os lodos pré-tratados que promoveram as maiores velocidades de produção de $\mathrm{H}_{2}$ foram utilizados para inocular reatores operados em batelada alimentada a $50^{\circ} \mathrm{C}$ com TRH de $1 \mathrm{~d}$. Os resultados da operação destes biorreatores por 36 dias pode ser observado na Figura 2. Como pode ser observado na Figura 2 o volume de $\mathrm{H}_{2}$ produzido pelos lodos pré-tratados foram bem mais elevados do que o volume de $\mathrm{H}_{2}$ produzido pelo lodo controle. $\mathrm{O}$ volume de $\mathrm{H}_{2}$ manteve-se praticamente constante ao longo do período de operação dos reatores inoculados com o lodo controle, $30 \mathrm{~mL}$. O volume de $\mathrm{H}_{2}$ produzido pelo lodo tratado por calor e ácido aumentou de 120 para 140 e $160 \mathrm{~mL} / \mathrm{d}$ ao final de 36 dias, respectivamente. 


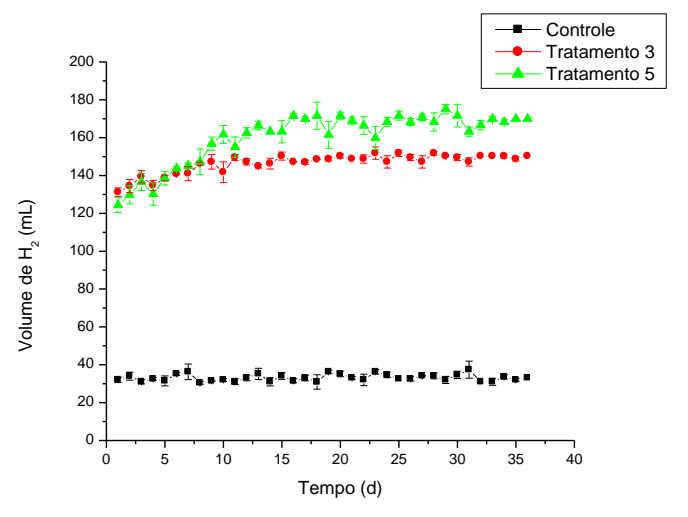

Figura 2 Volume de $\mathrm{H}_{2}$ produzido durante a operação dos biorreatores operados por 36 dias e inoculado com o lodo sem pré-tratamento (controle), pré-tratado a $80^{\circ} \mathrm{C} / 15 \mathrm{~min}$ (tratamento 3) e pré-tratado com $\mathrm{HCl}(\mathrm{pH} 3 / 24 \mathrm{~h})$.

Portanto, o pré-tratamento ácido do lodo foi o que promoveu o maior aumento na produção de $\mathrm{H}_{2}$ em relação ao controle. Luo et al., (2010) não observaram o mesmo efeito de pré-tratamentos comparando os resultados dos ensaios em batelada com os de operação por longos períodos. Os autores aplicaram diferentes pré-tratamentos em lodo anaeróbio, verificando em seguida o mais eficiente na produção de gás $\mathrm{H}_{2}$ a $60^{\circ} \mathrm{C}$. Após esta primeira etapa, realizada em batelada, os reatores foram operados em modo contínuo com TRH de 3 dias. Durante o estado estacionário de operação destes reatores foi observado que o lodo anaeróbio, sem qualquer tipo de pré-tratamento, exibiu a maior produção de $\mathrm{H}_{2}$, diferente dos resultados observados neste trabalho, no qual o efeito do aumento da produção de $\mathrm{H}_{2}$ pelo pré-tratamento do inóculo se manteve por um longo período.

Na Tabela 2 pode ser observada a concentração de metabólitos solúveis nos biorreatores, inoculados com os lodos pré-tratados e com o lodo controle, ao final do período de 36 dias de operação em batelada alimentada.

Tabela 2 Concentração de metabólitos solúveis nos biorreatores após 36 dias de operação.

\begin{tabular}{lcccccr}
\hline Inóculo & \multicolumn{7}{c}{ Metabólitos solúveis } \\
\cline { 2 - 7 } & $\begin{array}{l}\text { Ác. acético } \\
(\mathrm{mg} / \mathrm{L})\end{array}$ & $\begin{array}{l}\text { Ác.butírico } \\
(\mathrm{mg} / \mathrm{L})\end{array}$ & $\begin{array}{l}\text { Ác.propiônico } \\
(\mathrm{mg} / \mathrm{L})\end{array}$ & $\begin{array}{l}\text { Ác. lático } \\
(\mathrm{mg} / \mathrm{L})\end{array}$ & $\begin{array}{l}\text { Etanol } \\
(\mathrm{mg} / \mathrm{L})\end{array}$ & $\mathrm{Yp} / \mathrm{s}$ \\
\hline Controle & 0,61 & 0,57 & 0,58 & 9,0 & nd & 0,07 \\
\hline Térmico & 1,55 & 1,18 & 1,57 & 5,25 & nd & 1,1 \\
\hline Ácido & 1,51 & 2,90 & 0,24 & 2,0 & nd & 1,35 \\
\hline
\end{tabular}

nd: não detectado; Yp/s: $\mathrm{mol} \mathrm{H}_{2}$ produzido/mol glicose consumida

Como pode ser observado na Tabela 2 a concentração dos ácidos acético e butírico, metabólitos relacionados à produção de $\mathrm{H}_{2}$, foi mais elevada nos biorreatores inoculados com os lodos pré-tratados, o térmico e o ácido. Já o ácido lático foi detectado em maior concentração no lodo que não sofreu nenhum tipo de pré-tratamento (controle). A via bioquímica de formação do ácido lático em culturas mistas compete com a formação do $\mathrm{H}_{2}$, pois é o ácido lático consume equivalentes redutores, necessários à produção de $\mathrm{H}_{2}$. Portanto, pode-se afirmar que o pré-tratamento ácido foi o mais eficiente para o enriquecimento de bactérias produtoras de $\mathrm{H}_{2}$.a partir do inóculo estudado. 


\section{CONCLUSÕES}

Neste trabalho foi verificada a possibilidade de utilização de uma cultura mista proveniente de um sistema anaeróbio de tratamento de vinhaça na produção de $\mathrm{H}_{2}$. Os resultados revelaram que a cultura mista necessita de um pré-tratamento para ser utilizada como inóculo de biorreatores para a produção de $\mathrm{H}_{2}$. Dentre os pré-tratamentos testados concluiu-se que o tratamento ácido em $\mathrm{pH} 3$ por 24 horas foi o que, em testes de curta e longa duração, promoveu a maior produção de $\mathrm{H}_{2}$.

\section{REFERÊNCIAS BIBLIOGRÁFICAS}

APHA, AWWA, WEF.. Standard methods for the examination of water and wastewater. 19th. edn. American Public Health Association. Washington, DC, 1995.

BUITRÓN, G.; CARVAJAL, C. Biohydrogen production from Tequila vinasses in an anaerobic sequencingbatch reactor: Effect of initial substrate concentration, temperature and hydraulic retention time. Bioresource Technology, v.101, p. 9071-9077, 2010.

CHANG, S., LI, J.Z, LIU, F. Evaluation of different pretreatment methods for preparing hydrogen-producing seed inocula from waste activated sludge. Renewable Energy, v. 36, p. 1517-1522, 2011.

CHEN, C.C.; CHUANG, Y.S.; LIN, C.Y.; LAY, C.H.; SEM, B. Thermophilic dark fermentation of untreated rice straw using mixed cultures for hydrogen production. International Journal of Hydrogen Energy, v.37, p. 15540-15546, 2012.

DEL NERY, V. Utilização de lodo anaeróbio imobilizado em gel no estudo de partida de reatores de fluxo ascendente com manta de lodo, 215 p. Universidade de São Paulo, Escola de Engenharia de São Carlos, 1987.

LAY, C.H.; LIN,H.C., SEM, B.; CHU, C.Y.; LIN, C.Y. Simultaneous hydrogen and ethanol production from sweet potato via dark fermentation. Journal of Cleaner Production, v. 27, p. 155-164, 2012.

LUO, G.; XIE, L.; ZOU,,Z.; WANG,W.; ZHOU, Q. Fermentative hydrogen production from cassava stillage by mixed anaerobic microflora: Effects of temperature and $\mathrm{pH}$. Journal Applied Energy, v. 87, p. 3710-3717, 2010.

MOHAN, S.V. Harnessing of biohydrogen from wastewater treatment using mixed fermentative consortia: Process evaluation towards optimization. International Journal of Hydrogen Energy, v. 34, p. 7460-7474, 2009.

NING, Y. Y., JIN, D. W., SHENG, G. P., HARADA, H., SHI, X. Y., Evaluation of the stability of hydrogen production and microbial diversity by anaerobic sludge with chloroform treatment. Internacional. Journal Renewable Energy, v.38, p. 253-257, 2012. 